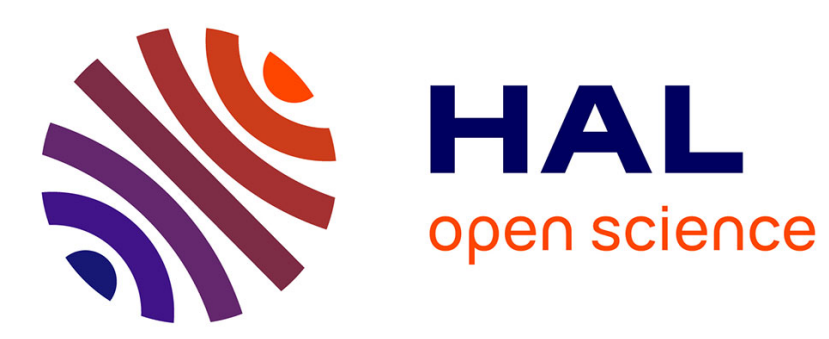

\title{
Control of cellular automata
}

Franco Bagnoli, Raúl Rechtman, Samira El Yacoubi

\section{To cite this version:}

Franco Bagnoli, Raúl Rechtman, Samira El Yacoubi. Control of cellular automata. Physical Review E: Statistical, Nonlinear, and Soft Matter Physics, 2012, 86 (6), pp.066201. 10.1103/PhysRevE.86.066201 . hal-03042120

\section{HAL Id: hal-03042120 https://hal-univ-perp.archives-ouvertes.fr/hal-03042120}

Submitted on 2 Jun 2021

HAL is a multi-disciplinary open access archive for the deposit and dissemination of scientific research documents, whether they are published or not. The documents may come from teaching and research institutions in France or abroad, or from public or private research centers.
L'archive ouverte pluridisciplinaire HAL, est destinée au dépôt et à la diffusion de documents scientifiques de niveau recherche, publiés ou non, émanant des établissements d'enseignement et de recherche français ou étrangers, des laboratoires publics ou privés. 


\title{
Control of cellular automata
}

\author{
Franco Bagnoli* \\ Dipartimento di Energetica, Università di Firenze, Via S. Marta 3 \\ I-50139 Firenze, Italy, also CSDC and INFN, sez. Firenze. \\ Raúl Rechtman ${ }^{\dagger}$ \\ Centro de Investigación en Energía, Universidad Nacional Autónoma de México, \\ Apdo. Postal 34, 62580 Temixco, Mor., Mexico. \\ Samira El Yacoubi \\ Institut de Modélisation et Analyse en Géo-Environnement et Santé (IMAGES), \\ Université de Perpignan, 52, Paul Alduy Avenue, 66860 - Perpignan Cedex. France..$^{\ddagger}$
}

(Dated: October 15, 2018)

\begin{abstract}
We study the problem of master-slave synchronization and control of totalistic cellular automata (CA) by putting a fraction of sites of the slave equal to those of the master and finding the distance between both as a function of this fraction. We present three control strategies that exploit local information about the CA, mainly, the number of nonzero Boolean derivatives. When no local information is used, we speak of synchronization. We find the critical properties of control and discuss the best control strategy compared with synchronization.
\end{abstract}

\section{INTRODUCTION}

There is a certain interest in modelling extended systems using discrete units, in many cases Boolean ones. There are two main areas for which discrete modelling is appropriate: systems whose dynamics are well approximated by a discrete process, and those for which discrete processes constitute a simplified computational description. Examples of systems of the first kind are genetic networks [1], some formalization of neural networks [2], DNA replication and translation [3], and VLSI digital circuits. These discrete models are stable and predictable in spite of a noisy environment. In order to overcome noisy fluctuations affecting signals of electronic devices, a large negative feedback is added, decreasing the overall efficiency but making the system more reliable. The extreme case of this approach is that of working at the saturation level of devices (e.g., transistors), leading to a digital representation of the problem. In biology, DNA replication and translation is essentially a digital process, and in many aspects gene control is also a kind of deterministic device, even in the presence of a fluctuating environment.

On the other hand, discrete modelling and in particular cellular automata are widely used as effective computational tools for simulating complex systems [4]. The idea is that of looking for the emergence of a complex dynamics out of an ensemble of simple components. Some examples are opinion formation, epidemics, and traffic $[5,6]$.

A discrete system is formed by separate units which can be nodes or sites. The state of each unit is taken

\footnotetext{
* franco.bagnoli@unifi.it

† rrs@cie.unam.mx

$\ddagger$ yacoubi@univ-perp.fr
}

from a discrete set and evolves in discrete time steps. Cellular automata (CA) [7] are the typical mathematical examples of such systems, even though one may be also interested in non-homogeneous contact networks and non-parallel dynamics [8].

We aim to study the dynamical properties of discrete dynamical systems, and in particular how these characteristics may be exploited for their control. In the following we shall consider simple one-dimensional CA, but most of our considerations can be extended to more complex systems.

The state of a discrete dynamical system is given by a discrete set of numbers, and a trajectory is simply given by a list of such states. Since the total number of possible states is finite, and due to the deterministic character of the system, after an eventual transient the trajectory has to enter a cyclic behavior (including fixed points). However, the size of this periodic attractor and the number of different attractors may scale with the size of the system. These numbers may grow exponentially with the system size, and quickly become intractable for practical purposes. We shall denote these as discrete extended systems.

Discrete extended systems are not chaotic in the usual sense. They have been selected or built with the explicit goal of being insensitive to small perturbations. However, they react to finite stimuli (change of state of some element), as in the experiments of gene knockout or silencing [9]. In this case, the reaction may be localized, or may extend to the whole system. In the latter case, the future evolution of the system is practically unpredictable. This is a situation analogous to usual chaotic systems, and the term "stable chaos" has been coined for such systems [10].

Although classical perturbation theory cannot be applied to these systems, it is possible to define the discrete analogous of derivatives (Boolean derivatives [11, 12]), as 
illustrated in Sec. II. By using this concept, it is then possible to construct a Jacobian matrix and to define the largest Lyapunov exponent, which discriminates well between systems that are "stable chaotic" and others whose evolution is more predictable [13].

A different, but complementary, approach to the study of the chaotic properties of a system is based on masterslave synchronization [14]. The idea is that of driving the slave with part of the signal from the master. It is possible to show that, for simple maps or for a uniform driving in extended continuous systems, there is a relationship between the synchronization threshold and the largest Lyapunov exponent.

A modified version of this synchronization procedure can be applied to discrete systems. The idea is that of synchronizing them using an all-or-nothing procedure; a fraction of sites are chosen at random and the state in the slave system at those sites, is set equal to that of of the corresponding sites in the master system (pinching synchronization) [13]. Applied to cellular automata, the pinching synchronization threshold shows a remarkable correlation with the previously defined largest Lyapunov exponent defined using Boolean derivatives.

The pinching synchronization procedure defines a directed percolation process among defects, that is, the sites that differ between the master and the slave. We say that the two replicas are not synchronized if in the long time limit there is some defect surviving (a more precise definition is given in the following section).

The synchronized state is absorbing, once it is reached, it cannot be abandoned. However, in the absence of a synchronization effort, the synchronized state is unstable for "chaotic" systems, since a finite perturbation will in general propagate to the rest of the system (as said, this is indeed a possible definition of a chaotic discrete system). The synchronization efforts (pinching synchronization) has the goal of making the synchronized state stable, and the synchronization transition corresponds to the marginal stability of this state.

By considering now only the points to which the pinching synchronization is not applied, one has a percolation backbone over which the defects can survive. Clearly, there is an upper value of the pinching synchronization probability over which the percolation backbone disappears and and synchronization takes place regardless of the rule. Differently from highly chaotic maps [15], all cellular automata synchronize well below such limit, indicating that the self-annihilation of defects plays a fundamental role in the synchronization transition. Indeed, the disappearance of defects is due both to the pinching procedure (which can be random and uncorrelated) and to the self-annihilation process, which in general is correlated. This correlation has a deep impact on the criticality of the phase transition, as we show in Section III.

We finally arrive to the main point of this paper. In control theory a controller is used to make a dynamical system behave in a specified way. The problem of control of a dynamical system may be split into two parts: the driving of the system to a target area in phase space, and the stabilization of a given trajectory originating from this area. Chaotic systems are ideal targets for control, since their sensitivity to small changes may be exploited to drive them to the target area [16]. After that driving phase, chaos may be suppressed in order to make them follow, for instance, a desired periodic orbit [17].

We shall concentrate on the second part, the stabilization of a given trajectory. Let us denote with the term "natural" a trajectory that is generated by the dynamics of the system, and with the term "artificial" all the other trajectories. In other words, if we initialize the given system in one of the states of a natural trajectory, then the system will follow the trajectory without any additional external control. On the contrary, "artificial" trajectories need a continuous control to force the system under study to follow them.

The problem of driving a chaotic system on a natural trajectory may be seen as the problem of synchronizing a "slave" with a "master" system that happens to follow the desired trajectory. However, while in the studies about synchronization one exerts little attention to the optimization of coupling, when formulated as a control problem this becomes a crucial issue.

In synchronization, the effort is applied at every site. In control problems, one wants to exploit some knowledge about the system. It is therefore analogous to a synchronization problem of two different systems with a "targeted" force, that tries to "kill" the defects in an efficient way. We show how the concept of Boolean derivatives can be exploited to achieve this goal [18].

The result is however rather surprising: the control efforts should concentrate on the regions giving birth to a small number of defects, while chaos can be exploited to "self-synchronize" the systems.

In Section II we present definitions of totalistic cellular automata, of Boolean derivatives, and synchronization and control of CA. Results of synchronization and three control strategies are discussed in Section III and in Section IV we present some final comments.

\section{CHAOS, SYNCHRONIZATION AND CONTROL OF CELLULAR AUTOMATA}

In this Section we recall the definition of totalistic Boolean cellular automata of range $R$, of Boolean derivatives, and present the master-slave synchronization and control of cellular automata [12, 13, 19].

A Boolean cellular automaton of range $R$ is a map $\boldsymbol{f}: \mathcal{B}^{N} \rightarrow \mathcal{B}^{N}$ with $\mathcal{B}=\{0,1\}, N$ large, and $\boldsymbol{f}=$ $\left(f_{0}, \ldots, f_{N-1}\right)$ with $f_{i}: \mathcal{B}^{N} \rightarrow \mathcal{B}, i=0, \ldots, N-1$. A state of the CA is $\boldsymbol{x}=\left(x_{0}, \ldots, x_{N-1}\right)$ with $x_{i} \in \mathcal{B}$, $i=0, \ldots, N-1$. The map $\boldsymbol{f}$ defines a flow on $\mathcal{B}^{N}$,

$$
\boldsymbol{x}(t+1)=\boldsymbol{f}(\boldsymbol{x}(t))
$$

with $t=0,1, \ldots$ In what follows $f_{i}=f, \forall i$. 
Let $\mathcal{N}_{i}$ be a neighborhood of site $i$ with $R$ sites and

$$
s_{i}=\sum_{j \in \mathcal{N}_{i}} x_{j},
$$

with $0 \leq s_{i} \leq R$. For totalistic CA of range $R$,

$$
x_{i}(t+1)=f\left(s_{i}(t)\right)
$$

with $f:\{0, \ldots, R\} \rightarrow \mathcal{B}$. There are $2^{R+1}$ different $\mathrm{CA}$ of range $R$, each one defined by a $(R+1)$-tuple $\left(y_{0}, \ldots, y_{R}\right)$ such that $y_{j}=1$ if the outcome of the CA is one when $s_{i}=j$ and $y_{j}=0$ otherwise. In what folows, we use Vichniac's notation $R T C$, with $R$ the range, $T$ is for totalistic, and $C$ the number in base 10 of $\left(y_{0}, \ldots, y_{R}\right)$ [11].

The control mechanisms we consider depend on the knowledge of the local expanding properties of the CA given by the Boolean derivatives [12] defined by

$$
\begin{aligned}
J_{i, j}= & \frac{\partial f_{i}(\boldsymbol{x})}{\partial x_{j}} \\
= & f_{i}\left(x_{0}, \ldots, x_{j} \oplus 1, \ldots, x_{N-1}\right) \oplus \\
& f_{i}\left(x_{0}, \ldots, x_{j}, \ldots, x_{N-1}\right) \\
& = \begin{cases}1 & f_{i} \text { changes when } x_{j} \text { changes } \\
0 & f_{i} \text { does not change when } x_{j} \text { changes }\end{cases}
\end{aligned}
$$

with $\oplus$ the logical exclusive disjunction or what is the same, the sum modulo 2.

Higher order Boolean derivatives can be defined in a similar way and we can write any function $f$ of range $R$ in the equivalent of a Taylor or Maclaurin expansion that is exact if the expansion goes up to $R$-th order derivatives [12]. The exact MacLaurin expansion of a CA with $R=2$ is

$$
\begin{aligned}
f(x, y)= & \left.f(0,0) \oplus \frac{\partial f}{\partial x}\right|_{(0,0)} \cdot x \oplus \\
& \left.\left.\frac{\partial f}{\partial y}\right|_{(0,0)} \cdot y \oplus \frac{\partial^{2} f}{\partial x \partial y}\right|_{(0,0)} \cdot x \cdot y
\end{aligned}
$$

with - the logical conjunction. If the expansion of $f$ contains only the first order Boolean derivatives, we say that $f$ is linear. As an example, let $R=3, \mathcal{N}_{i}=$ $\left\{x_{i-1}, x_{i}, x_{i+1}\right\}$, and $f\left(s_{i}\right)=1$ if $s_{i}$ is odd and zero otherwise. This is the $3 T 10 \mathrm{CA}$ shown in Table I which is linear since

$$
f\left(x_{i-1}, x_{i}, x_{i+1}\right)=x_{i-1} \oplus x_{i} \oplus x_{i+1} .
$$

In Wolfram's notation, this is rule 150 [20]. Another example with $R=3$ is the $3 T 6 \mathrm{CA}$, rule 126 in Wolfram's notation, defined in such a way that $f\left(s_{i}\right)=1$ if $s_{i}=1,2$ and zero otherwise shown in Table II. It is nonlinear since

$$
\begin{aligned}
f\left(x_{i-1}, x_{i}, x_{i+1}\right)= & x_{i-1} \oplus x_{i} \oplus x_{i+1} \oplus \\
& x_{i-1} x_{i} \oplus x_{i-1} x_{i+1} \oplus x_{i} x_{i+1} .
\end{aligned}
$$

TABLE I. Truth table and Boolean derivatives of the $3 T 10$ CA.

\begin{tabular}{|ccc|c|c|c|c|c|}
\hline$x_{i-1}$ & $x_{i}$ & $x_{i+1}$ & $s_{i}$ & $f$ & $\partial f / \partial x_{i-1}$ & $\partial f / \partial x_{i}$ & $\partial f / \partial x_{i+1}$ \\
\hline 0 & 0 & 0 & 0 & 0 & 1 & 1 & 1 \\
0 & 0 & 1 & 1 & 1 & 1 & 1 & 1 \\
0 & 1 & 0 & 1 & 1 & 1 & 1 & 1 \\
0 & 1 & 1 & 2 & 0 & 1 & 1 & 1 \\
1 & 0 & 0 & 1 & 1 & 1 & 1 & 1 \\
1 & 0 & 1 & 2 & 0 & 1 & 1 & 1 \\
1 & 1 & 0 & 2 & 0 & 1 & 1 & 1 \\
1 & 1 & 1 & 3 & 1 & 1 & 1 & 1 \\
\hline
\end{tabular}

TABLE II. Truth table and Boolean derivatives of the $3 T 6$ CA.

\begin{tabular}{|ccc|c|c|c|c|c|}
\hline$x_{i-1}$ & $x_{i}$ & $x_{i+1}$ & $s_{i}$ & $f$ & $\partial f / \partial x_{i-1}$ & $\partial f / \partial x_{i}$ & $\partial f / \partial x_{i+1}$ \\
\hline 0 & 0 & 0 & 0 & 0 & 1 & 1 & 1 \\
0 & 0 & 1 & 1 & 1 & 0 & 0 & 1 \\
0 & 1 & 0 & 1 & 1 & 0 & 1 & 0 \\
0 & 1 & 1 & 2 & 1 & 1 & 0 & 0 \\
1 & 0 & 0 & 1 & 1 & 1 & 0 & 0 \\
1 & 0 & 1 & 2 & 1 & 0 & 1 & 0 \\
1 & 1 & 0 & 2 & 1 & 0 & 0 & 1 \\
1 & 1 & 1 & 3 & 0 & 1 & 1 & 1 \\
\hline
\end{tabular}

We now discuss a master-slave synchronization and control mechanism of cellular automata where the configuration $\boldsymbol{x}$ represents the master and $\boldsymbol{y}$ the slave. Let $p_{i}, i=0, \ldots, N-1$ be a Boolean variable that is 1 with probability $\pi_{i}$ and 0 otherwise. That is,

$$
p_{i}= \begin{cases}1 & \text { with probability } \pi_{i} \\ 0 & \text { with probability } 1-\pi_{i} .\end{cases}
$$

Then

$$
\begin{aligned}
x_{i}(t+1) & =f(\boldsymbol{x}(t)), \\
y_{i}(t+1) & =\left(1-p_{i}(t)\right) \cdot f(\boldsymbol{y}(t)) \oplus p_{i}(t) \cdot f(\boldsymbol{x}(t)), \\
& = \begin{cases}f(\boldsymbol{x}(t)) & \text { if } p_{i}(t)=1 \\
f(\boldsymbol{y}(t)) & \text { if } p_{i}(t)=0,\end{cases} \\
h_{i}(t+1) & =y_{i}(t+1) \oplus x_{i}(t+1) .
\end{aligned}
$$

In the last Eq. $h_{i}=1$ if $y_{i}$ is different from $x_{i}$ and is zero otherwise. We use the shorthand $\boldsymbol{h}=\boldsymbol{y} \oplus \boldsymbol{x}$. Wherever $p_{i}=1, y_{i}=x_{i}$ and we say that $\boldsymbol{y}$ and $\boldsymbol{x}$ are "pinched" at site $i$. We define the control parameter $k$ and the order parameter $h$ by

$$
k=\frac{1}{N} \sum_{i} p_{i}, \quad h=\frac{1}{N} \sum_{i} h_{i} .
$$

If $k=0, \boldsymbol{y}$ evolves independently of $\boldsymbol{x}$ and $h \neq 0$, even though the self-annihilation of defects is still present due to the Boolean character of the system. If $k=1, h=0$, 
(a)

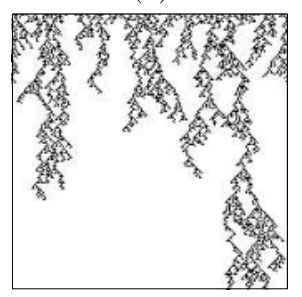

(b)

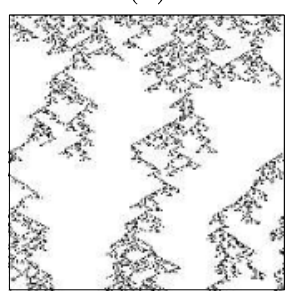

FIG. 1. Time evolution of the difference $\boldsymbol{h}$ for (a) CA $3 T 10$, (b) CA $5 T 42$ with $N=200$ during 200 time steps. Time runs from top to bottom.

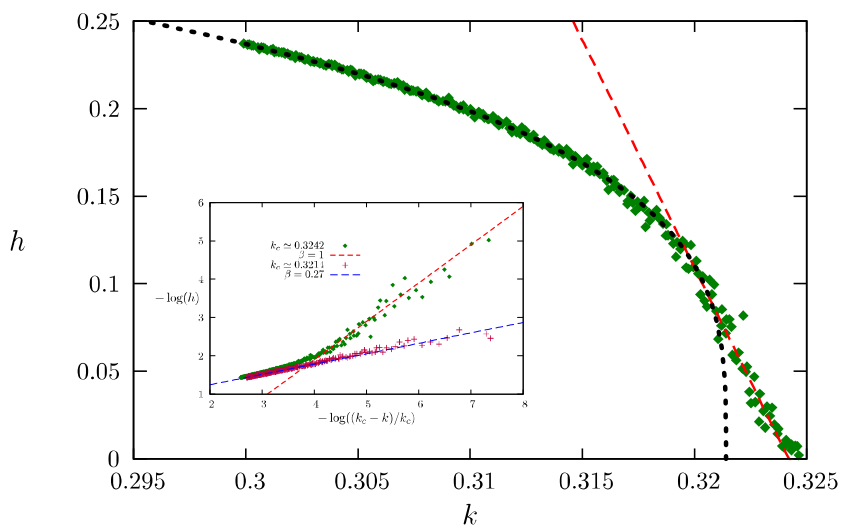

FIG. 2. The scaling of the asymptotic distance $h$ as a function of the synchronization effort $k$ for rule $3 \mathrm{~T} 10$ in case $\mathbf{0}$ and $N=20,000$. The curves follow the scaling law Eq. (8). The dotted curve corresponds to $\beta=0.27\left(k_{c} \simeq 0.321\right.$ ), compatible with directed percolation and the dashed line corresponds to $\beta=1\left(k_{c} \simeq 0.3275\right)$ compatible with a mean field behavior. In the inset, the scaling behavior is reported on a log-log scale.

both states are pinched together. There is a threshold $k_{c}$ above which $h=0$ in the long time limit. See Fig. 1 for a visual illustration of the synchronization process. If no knowledge is assumed about the local behavior of $f$ that defines the CA, we speak of pinching synchronization [13] and take $\pi_{i}=p$ for all $i$. In the case where some knowledge about $f$ is used to determine $\pi_{i}$ (that can change in time), we speak of pinching control.

In the following Sec. we show results for synchronization and three types of control based on the local expanding properties of the map $f$. We define $J_{i}$ as the sum of the $R$ Boolean derivatives that are affected by site $i$

$$
J_{i}=\sum_{j \in \mathcal{N}_{i}} J_{j, i}
$$

with $0 \leq J_{i} \leq R$ (notice the indices). The cases we consider are

0. $\pi_{i}(t)=p$ with $0 \leq p \leq 1$ independently of $i$ and $t$.

1. $\pi_{i}(t)$ is proportional to $J_{i}(t)$.

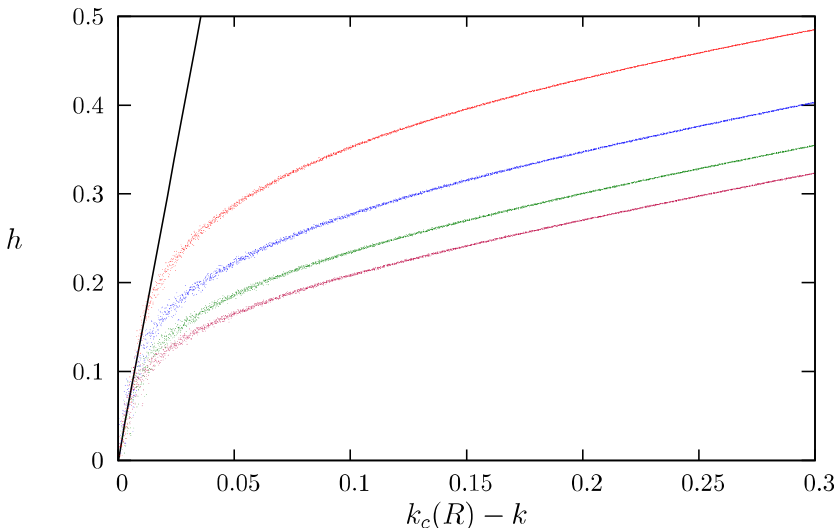

FIG. 3. Synchronization (control type 0) of some linear cellular automata, from top to bottom: $3 T 10,5 T 42,7 T 170$, $9 T 682$, rescaled to make the critical point coincide with the origin of axis. In these simulations $N=4,000$.

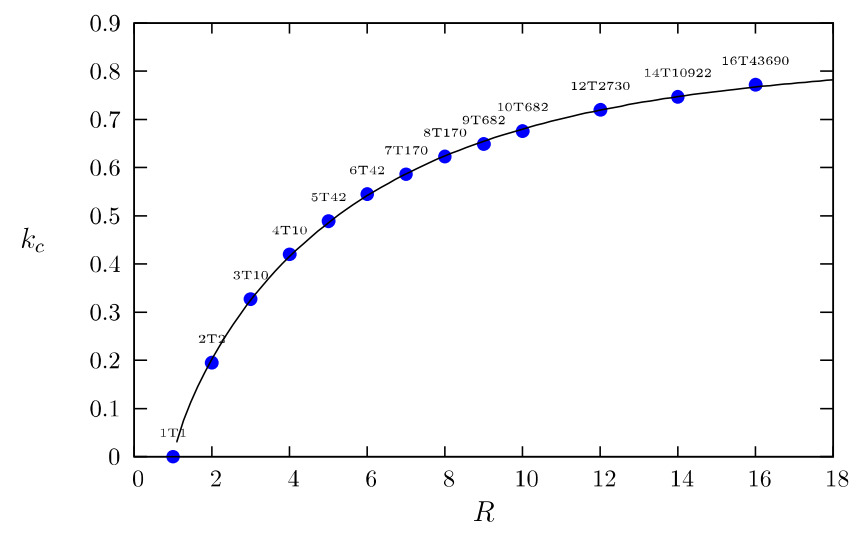

FIG. 4. The relation between $k_{c}$, and the range $R$ for linear cellular automata, control type $\mathbf{0}$. The solid line marks a the best fit of Eq. (??) with $k_{\infty}=0.825, \gamma=0.282$, and $\alpha=0.828$.

2. If $J_{i}(t)=0, \pi_{i}(t)=0$, otherwise $\pi_{i}(t)$ is proportional to $R-J_{i}(t)+1$

3. If $J_{i}(t)=0, \pi_{i}(t)=0$, otherwise $\pi_{i}(t)$ is proportional to $\left(R-J_{i}(t)+1\right)^{2}$.

Case $\mathbf{0}$ corresponds to synchronization, no knowledge on the dynamics of the CA is used. The other cases, use the derivatives in one way or the other. Since $0 \leq J_{i} \leq R$, case 1 will favor that states $\boldsymbol{x}$ and $\boldsymbol{y}$ be pinched at site $i$ at time $t$ when $J_{i}(t)$ is large. On the other hand, cases $\mathbf{2}$ and $\mathbf{3}$ favor pinching at site $i$ at time $t$ whenever $J_{i}(t)$ is small (case $\mathbf{3}$ more than case $\mathbf{2}$ ).

\section{RESULTS}

We begin by presenting the synchronization and control of linear CA. All types of control coincide with the 


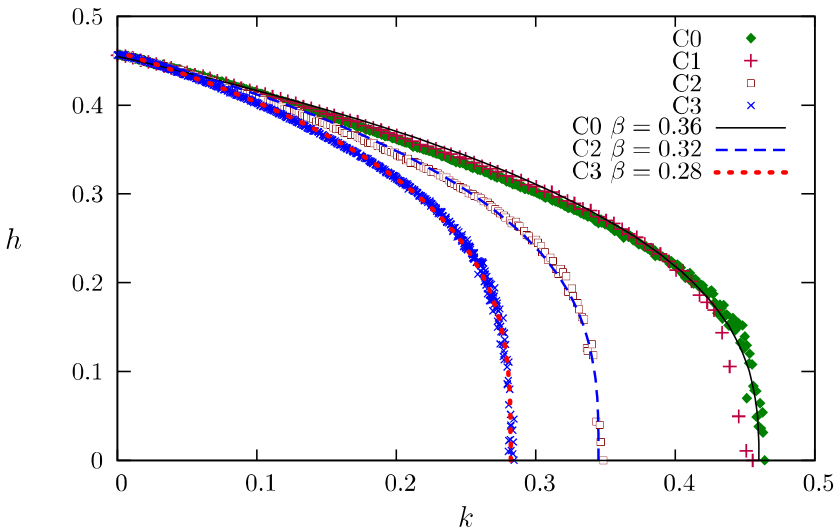

FIG. 5. Critical behavior of nonlinear CA $7 \mathrm{~T} 10$ for control types $\mathbf{0}, \ldots, \mathbf{3}$. In all cases Eq. (8) fits the data with $h_{0} \simeq 0.46$ and different values of $k_{c}$ and $\beta$ (continuous curves). Control type $\mathbf{0}$ and $\mathbf{1}$ agree numerically with $\beta \simeq 0.36$, control type $\mathbf{2}$ with $\beta=0.32$ control type 3 with $\beta=0.28$.

synchronization case $\mathbf{0}$ for linear $\mathrm{CA}$, since $J_{i}=R$ for every site $i$.

Near $k_{c}, h$ follows the scaling law

$$
h=h_{0}\left(\frac{k_{c}-k}{k_{c}}\right)^{\beta},
$$

with $h_{0}$ the average number of active sites for the unperturbed rule $(k=0)$.

In Fig. 2 we show the critical behavior of $h$ for CA $3 T 10$ that has a crossover between the directed percolation ( $\mathrm{DP}, \beta \simeq 0.27)$ and the mean field $(\mathrm{MF}, \beta=1$ ) universality classes. As we show in Fig. 3, other linear rules exhibit the same type of crossover from the MF to the DP universality class. As $R$ grows, the MF character of the transition occupies a smaller region. This behavior is rather unexpected, since the Grassberger-Jensen conjecture [21] should hold in this case. The interactions are short-range and, since the visual behavior is irregular, correlations are expected to be short-range. By applying a transformation $y=1 \oplus x$ for odd neighbourhoods one can show that for a linear CA, $h_{0}=1 / 2$.

In Fig. 4 we show the synchronization threshold $k_{c}$ of linear totalistic CAs as a function of the range $R$. The numerical data are well fitted by

$$
k_{c}=k_{\infty}\left(1-\exp \left(-\gamma(R-1)^{\alpha}\right)\right) .
$$

We assumed that for $R=1$ the critical value of $k_{c}$ is zero. In this case each site only has one neighbor, and therefore any infinitesimal control is able to synchronize the replicas in the long time limit. When $R \rightarrow \infty, k_{\infty} \simeq$ 0.825 .

For the nonlinear CA 7T10, the scaling behavior near the transition is more similar to a DP character, for all types of control, as shown in Fig. 5, even though the measured exponents differ numerically from the DP exponent.

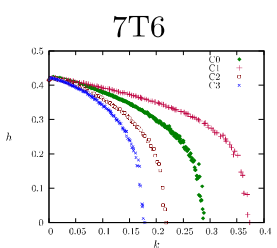

$6 \mathrm{~T} 60$

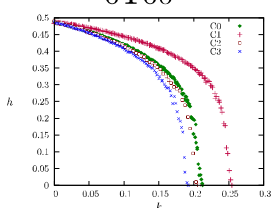

$5 \mathrm{~T} 45$
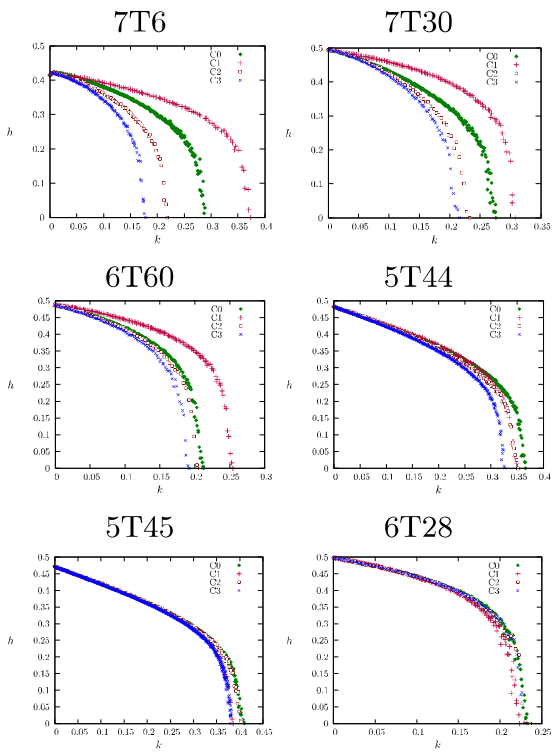

$5 \mathrm{~T} 44$

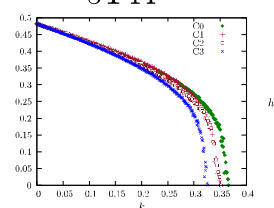

$6 \mathrm{~T} 28$

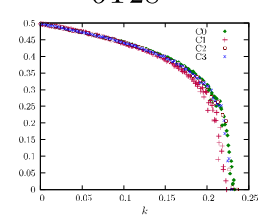

$3 \mathrm{~T} 6$

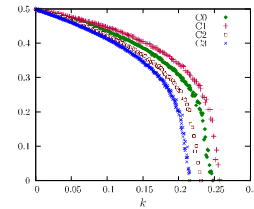

$5 \mathrm{~T} 46$

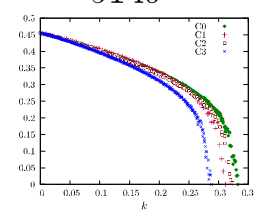

$7 \mathrm{~T} 60$

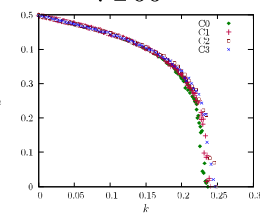

FIG. 6. Phase diagram of several nonlinear CA with $N=$ 4,000 .

For linear CA, all control cases are equivalent. This is not the case for nonlinear CA. Although we have not performed an exhaustive search, some nonlinear chaotic CA show the general pattern $k_{c}^{(3)} \leq k_{c}^{(2)} \leq k_{c}^{(0)} \leq k_{c}^{(1)}$ with $k_{c}^{(n)}$ the critical value of the control parameter $k$ in case $n, n=\mathbf{0}, \ldots, \mathbf{3}$. Examples are CA 7T6, 7T30, and $3 T 6$ whose critical behavior is shown in the top three graphs of Fig. 5. For the CA of the second line of Fig. 5 control 3 shows the smallest $k_{c}$. In the last line of Fig. 5 we show three nonlinear CA for for which the four types of control are similar.

In a large sample of nonlinear CA studied, that includes those of the previous Fig., the application of control 3 gives the smallest value of $k_{c}$ (which may coincide with the other types of control). A possible explanation is that, due to the irregular behavior of the $\mathrm{CA}$ and the presence of an absorbing state (the synchronized or controlled state), the system will approach this absorbing state in small neighborhhoods that can grow at most linearly in time. Thus, it is sufficient to break down the system into small portions, and for doing this it is more efficient to synchronize sites with a small (but non-zero) number of derivatives, which are presumably on the border of the non-synchronized patches, than synchronizing other sites in the bulk. Control $\mathbf{2}$ and even more control 3, preferably select sites with few non-zero derivatives, and are therefore more efficient. On the other hand, it is almost useless to synchronize sites in the bulk of the patches, with many derivatives, since they do not contribute much to the spreading of the desynchronized state.

This is not the whole story. As we show in Fig. 6, there are CA for which control type $\mathbf{3}$ is not the best one, even if the differences are minimal. At present, we have no 
explanation for this behavior.

\section{CONCLUSIONS}

We have studied the problem of master-slave synchronization and control of totalistic Boolean cellular automata via a pinching (all-or-none) mechanism. We have investigated four different cases: 0 pinching synchronization, $\mathbf{1}$ control proportional to the number of Boolean derivatives at a given site, and $\mathbf{2}$ and $\mathbf{3}$ controls for which the probability of applying the synchronization procedure diminishes with the number of Boolean derivatives. The number of nonzero local derivatives is an indicator of the expected number of defects.

For linear rules, the four types of control are equivalent and the control transition shows a crossover from a di- rected percolation universality class to a mean field one. More research is needed to decide this, since It is well known that the estimation of the exponent $\beta$ requires sophisticated techniques which are left for future study.

Nonlinear CA show a counter intuitive behavior. For some of them, control $\mathbf{3}$ is the most efficient followed by controls 2, $\mathbf{0}$, and $\mathbf{1}$, although there are CA for which this is not true. A more exhaustive research is needed in order to find some way to group CA according to the order in which they can be controlled.

\section{ACKNOWLEDGEMENTS}

Interesting discussions on control of extended systems with A. El Jai are acknowledged. Economic support for part of this work from project 25116 CONACyT-Mexico is als acknowledged.
[1] S.A. Kauffman, Metabolic stability and epigenesis in randomly constructed genetic nets". J. Theor. Biol. 22, 437467 (1969). For a recent application see: C Damiani, R. Serra, M. Villani, S.A. Kauffman and A. Colacci, Cellcell interaction and diversity of emergent behaviours, IET Syst. Biol. 5, 137-44 (2011).

[2] J. Chorowski and J.M. Zurada, Extracting Rules from Neural Networks as Decision Diagrams, IEEE Trans Neural Netw, Feb 17 [Epub ahead of print] (2011).

[3] See any textbook in molecular biology, for instance $H$. Lodish, A. Berk, S Lawrence Zipursky, P. Matsudaira, D. Baltimore, and J. Darnell, Molecular Cell Biology, 4th edition (W. H. Freeman, New York, 2000).

[4] See the series of proceeding of the conference ACRI: Cellular Automata for Research and Industry published in Lectures Notes in Computer Science (Springer) 6350 (2010) 5191 (2008) $\mathbf{4 1 7 3}$ (2006) $\mathbf{3 3 0 5}$ (2004) 2493 (2002).

[5] B. Chopard Bastien and M. Droz, Cellular Automata Modeling of Physical Systems (Cambridge University Press, Cambrige UK, 2005).

[6] A. Hoekstra, J. Kroc, P. Sloot, (Eds.) Simulating Complex Systems by Cellular Automata (Springer, Berlin 2010.

[7] B. Chopard and M. Droz, Cellular Automata Modeling of Physical Systems (Cambridge University Press, 1998); J.L. Schiff, Cellular Automata: A Discrete View of the World ISBN 047016879X (Wiley \& Sons, Inc., 2002).

[8] A. Graudenzi, R. Serra, M. Villani, C. Damiani, A. Colacci, S.A. Kauffman, Dynamical properties of a boolean model of gene regulatory network with memory, J. Comput. Biol. 18, 1291-303 (2011).

[9] There are many techniques of gene inactivation, silencing or removal, a good starting point is a modern book in genetic engineering like S. B. Primrose and R. Twyman, Principles of Gene Manipulation and Genomics, ISBN
978-1-4051-3544-3 (Wiley-Blackwell, 2006).

[10] J.P. Crutchfield and K. Kaneko, Phys. Rev. Lett. 60, 2715 (1988); K. Kaneko, Phys. Lett. 149A, 105 (1990) A. Politi, R. Livi, G.-L. Oppo, and R. Kapral, Europhys. Lett. 22, 571 (1993); F. Bagnoli and F. Cecconi, Phys. Lett. A 260, 9-17 (2001).

[11] G. Vichniac, Physica 10D 96 (1984).

[12] F. Bagnoli, Int. J. Mod. Phys. C. 3307 (1992).

[13] F. Bagnoli, R. Rechtman, Phys. Rev. E 59 R1307 (1999).

[14] L.M. Pecora and T.L. Carroll, Phys. Rev. Lett. 64, 821 (1990).

[15] F. Bagnoli, L. Baroni and P. Palmerini, Phys. Rev. E 59, 409 (1999)

[16] T. Shinbrot, E. Ott, C. Grebogy and J.A. Yorke, Phys. Rev. Lett. 65, 3215 (1990).

[17] E. Ott, C. Grebogy and J.A. Yorke, Phys. Rev. Lett. 64, 1196 (1990).

[18] F. Bagnoli, S. El Yacoubi, R. Rechtman, Synchronization and control of cellular automata, Cellular Automata, $9^{t h}$ International Conference on Cellular Automata for Research and Industry, ACRI 2010, Ascoli Piceno, Italy, September 21-24 2010, Lecture Notes in Computer Science, S. Bandini, S. Manzoni, H. Umeo, G. Vizzari (eds.), 6350, 188 (2010), Springer.

[19] F. Bagnoli, R. Rechtman, S. Ruffo, Phys. Lett. A 17234 (1992).

[20] S. Wolfram, Rev. Mod. Phys. 55601 (1983).

[21] H. K. Janssen, On the nonequilibrium phase transition in reaction-diffusion systems with an absorbing stationary state, Z. Phys. B 42, 151154 (1981). P. Grassberger, On phase transitions in Schlögl's second model, Z. Phys. B 47, 365374 (1982).

H. Hinrichsen, Nonequilibrium Critical Phenomena and Phase Transitions into Absorbing States, Adv. Phys. 49 815-958 (2000). 